Article

\title{
Engineering Bafilomycin High-Producers by Manipulating Regulatory and Biosynthetic Genes in the Marine Bacterium Streptomyces lohii
}

\author{
Zhong Li ${ }^{1,2,3}$, Shuai Li ${ }^{1}$, Lei Du ${ }^{1} \mathbb{D}$, Xingwang Zhang ${ }^{1}$, Yuanyuan Jiang ${ }^{1,2,3}$, Wenhua Liu ${ }^{1}$, Wei Zhang ${ }^{1}$ \\ and Shengying $\mathrm{Li}^{1,4, * \mathbb{D}}$
}

1 State Key Laboratory of Microbial Technology, Shandong University, Qingdao 266237, China; lizhong@qibebt.ac.cn (Z.L.); sdu_lishuai@mail.sdu.edu.cn (S.L.); lei.du@sdu.edu.cn (L.D.); zhangxingwang@sdu.edu.cn (X.Z.); jiangyy@qibebt.ac.cn (Y.J.); liuwh@sdu.edu.cn (W.L.); zhang_wei@sdu.edu.cn (W.Z.)

2 Shandong Provincial Key Laboratory of Synthetic Biology, CAS Key Laboratory of Biofuels at Qingdao Institute of Bioenergy and Bioprocess Technology, Chinese Academy of Sciences, Qingdao 266101, China

3 College of Life Sciences, University of Chinese Academy of Sciences, Beijing 100049, China

4 Laboratory for Marine Biology and Biotechnology, Qingdao National Laboratory for Marine Science and Technology, Qingdao 266237, China

* Correspondence: lishengying@sdu.edu.cn

check for

updates

Citation: Li, Z.; Li, S.; Du, L.; Zhang, X.; Jiang, Y.; Liu, W.; Zhang, W.; Li, S. Engineering Bafilomycin

High-Producers by Manipulating Regulatory and Biosynthetic Genes in the Marine Bacterium Streptomyces lohii. Mar. Drugs 2021, 19, 29. https://doi.org/md19010029

Received: 2 December 2020 Accepted: 8 January 2021 Published: 11 January 2021

Publisher's Note: MDPI stays neutral with regard to jurisdictional clai$\mathrm{ms}$ in published maps and institutional affiliations.

Copyright: (C) 2021 by the authors. Licensee MDPI, Basel, Switzerland. This article is an open access article distributed under the terms and conditions of the Creative Commons Attribution (CC BY) license (https:// creativecommons.org/licenses/by/ $4.0 /)$.

\begin{abstract}
Bafilomycin $A_{1}$ is the representative compound of the plecomacrolide natural product family. This 16-membered ring plecomacrolide has potent antifungal and vacuolar $\mathrm{H}^{+}$-ATPase inhibitory activities. In our previous work, we identified a bafilomycin biosynthetic gene cluster (baf) from the marine bacterium Streptomyces lohii ATCC BAA-1276, wherein a luxR family regulatory gene orf1 and an afsR family regulatory gene $b a f G$ were revealed based on bioinformatics analysis. In this study, the positive regulatory roles of orf1 and bafG for bafilomycin biosynthesis are characterized through gene inactivation and overexpression. Compared to the wild-type S. lohii strain, the knockout of either orf1 or bafG completely abolished the production of bafilomycins. The overexpression of orf1 or bafG led to 1.3- and 0.5-fold increased production of bafilomycins, respectively. A genetically engineered S. lohii strain (SLO-08) with orf1 overexpression and inactivation of the biosynthetic genes orf 2 and orf 3 , solely produced bafilomycin $\mathrm{A}_{1}$ with the titer of $535.1 \pm 25.0 \mathrm{mg} / \mathrm{L}$ in an optimized fermentation medium in shaking flasks. This recombinant strain holds considerable application potential in large-scale production of bafilomycin $\mathrm{A}_{1}$ for new drug development.
\end{abstract}

Keywords: Streptomyces; bafilomycin; regulatory gene; biosynthesis; fermentation optimization

\section{Introduction}

Bafilomycins, which are mainly produced by Streptomyces, belong to the plecomacrolide (i.e., a 16- or 18-membered macrolactone connected to a 6-membered hemiacetal ring via a three-carbon linker) subfamily of polyketide natural products. These 16-membered ring macrolides have shown diverse biological activities including antibacterial [1], antifungal [2], antitumor [3], and anti-osteoporotic [4] activities. Since bafilomycin $\mathrm{A}_{1}, \mathrm{~B}_{1}$, and $C_{1}$ were first isolated from Streptomyces griseus sp. sulphurus (TÜ 1922) in 1983 [5], nearly thirty bafilomycin derivatives have been discovered [5-7]. Essentially, most of these derivatives (Figure 1) are generated from the bafilomycin $\mathrm{A}_{1}$ core structure through various known [8-10] and unknown tailoring steps during their biosynthetic pathways.

As the first and archetypal compound of bafilomycins, bafilomycin $\mathrm{A}_{1}$ has attracted much attention because it is a potent and specific inhibitor of vacuolar $\mathrm{H}^{+}$-ATPase (VATPase), which is an important drug target for osteoporosis [4]. This compound may also be applied in antitumor therapy due to its potent autophagy inhibitory activity, which prevents autophagosome-lysosome fusion in cells by targeting the sarco/endoplasmic reticulum 
$\mathrm{Ca}^{2+}$-ATPase (SERCA) pump [11]. Moreover, bafilomycin $\mathrm{A}_{1}$ has been demonstrated to show promising prospects in the field of combined pharmacotherapy. For example, bafilomycin $\mathrm{A}_{1}$ and FK506 have displayed marked synergistic antifungal activities against the fungal pathogen Cryptococcus neoformans [12]; the combined treatment with bortezomib plus bafilomycin $A_{1}$ has been proved to be capable of enhancing the cytocidal effect and inducing U266 myeloma cells [13]; and the inhibition of autophagy by bafilomycin $A_{1}$ can decrease the resistance of gastric cancer cells to 5-fluorouracil in vitro [14]. Intriguingly, bafilomycin $A_{1}$ was recently reported to be capable of interrupting the function of the viral receptor ACE2 via inhibiting the V-ATPase, thus being considered as a candidate for treating the infections caused by coronaviruses (e.g., COVID-19, SARS-CoV, and MERS$\mathrm{CoV}$ ) [15]. Despite these promising results, such a potent drug candidate has not entered clinical application owing to its high toxicity to mammalian cells [16]. Thus, bafilomycin $\mathrm{A}_{1}$ has become an attractive target for medicinal chemists to synthesize unnatural bafilomycin derivatives with lower toxicity [17]. For the purposes of new drug development and diverse bioactivity assays, the demands for bafilomycin $A_{1}$ are fast growing, which has naturally led to the demand for bafilomycin $\mathrm{A}_{1}$ high-producing strains since total synthesis of bafilomycin $A_{1}$ remains highly challenging $[18,19]$.

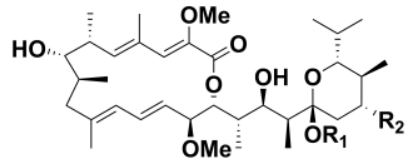

Bafilomycin $A_{1} R_{1}=H, R_{2}=O H$; Bafilomycin $A_{2} R_{1}=M e, R_{2}=O H$ 21-Deoxybafilomycin $A_{1} \quad R_{1}=H, R_{2}=H$

21-Deoxybafilomycin $A_{2} \quad R_{1}=M e, R_{2}=H$

Bafilomycin $G R_{1}=H, R_{2}=O M e ;$ Bafilomycin $H R_{1}=M e, R_{2}=O M e$

HO...

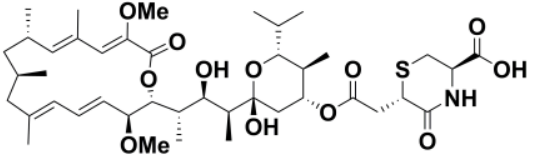

Bafilomycin F

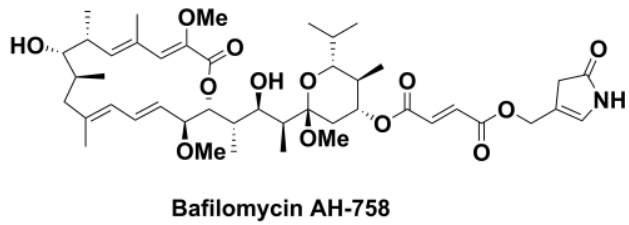

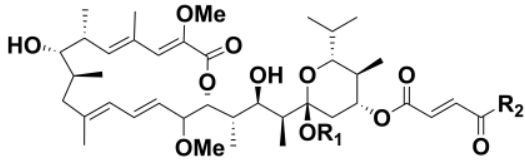

Bafilomycin $\mathrm{C}_{1} \mathrm{R}_{\mathbf{1}}=\mathrm{H}, \mathrm{R}_{\mathbf{2}}=\mathrm{OH}$

Bafilomycin $\mathrm{C}_{2} \mathrm{R}_{\mathbf{1}}=\mathrm{Me}, \mathrm{R}_{\mathbf{2}}=\mathrm{OH}$

Bafilomycin $\mathrm{C}_{1}$ amide $\mathrm{R}_{1}=\mathrm{H}, \mathrm{R}_{\mathbf{2}}=\mathrm{NH}_{2}$

Bafilomycin $\mathrm{C}_{2}$ amide $\mathrm{R}_{1}=\mathrm{Me}, \mathrm{R}_{2}=\mathrm{NH}_{2}$

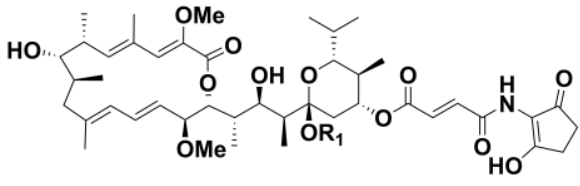

Bafilomycin $\mathrm{B}_{1} \mathrm{R}_{1}=\mathrm{H}$; Bafilomycin $\mathrm{B}_{2} \mathrm{R}_{1}=\mathrm{Me}$

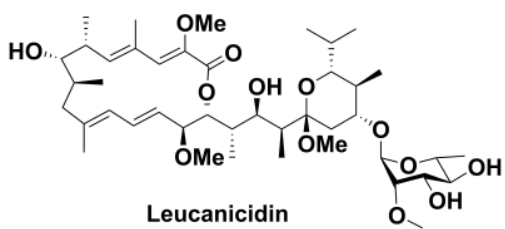

Figure 1. Structures of representative bafilomycins.

To engineer a bafilomycin $A_{1}$ high-producer, knowledge on its biosynthetic mechanisms is required. To date, at least six bafilomycin biosynthetic gene clusters from Streptomyces and Kitasatospora species have been reported by this and other laboratories $[8,10,20-23]$. The modular and domain organization in five type I polyketide synthase (PKS) genes (exemplified by bafAI-bafAV, Figure $2 a$ ) collinearly matches the structure of bafilomycin $\mathrm{A}_{1}$. The five open reading frames $b a f B-F$ are responsible for biosynthesis of the methoxymalonate extender unit based on bioinformatics analysis. Recently, we completely elucidated the post-PKS tailoring steps (Figure $2 b$ ) of the bafilomycin biosynthetic pathway in Streptomyces lohii ATCC BAA-1276. Specifically, the adenylyltransferase Orf3 activates fumarate to fumaryl-AMP, whose fumaryl moiety is then transferred to C21-hydoxyl group of bafilomycin $A_{1}$ by the fumaryl transferase Orf2, giving rise to bafilomycin $C_{1}$. Next, the ATP-dependent amino synthetase BafY catalyzes the $\mathrm{C}-\mathrm{N}$ bond formation between bafilomycin $C_{1}$ and 2-amino-3-hydroxycyclopent-2-enone $\left(C_{5} N\right)$ to form bafilomycin $B_{1}$. The $\mathrm{C}_{5} \mathrm{~N}$ unit is assembled by the acyl-CoA ligase BafX and the bifunctional enzyme BafZ $[9,20]$. 
(a)

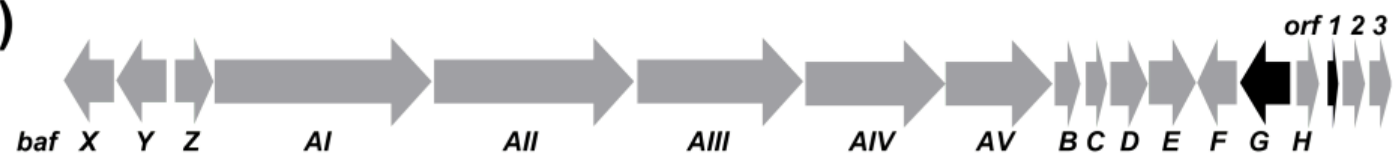

(b)

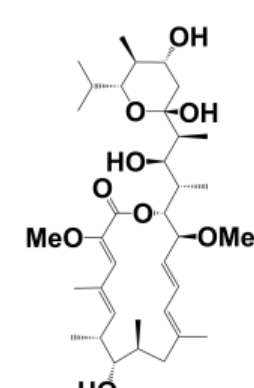

HO

Bafilomycin $\mathbf{A}_{1}$

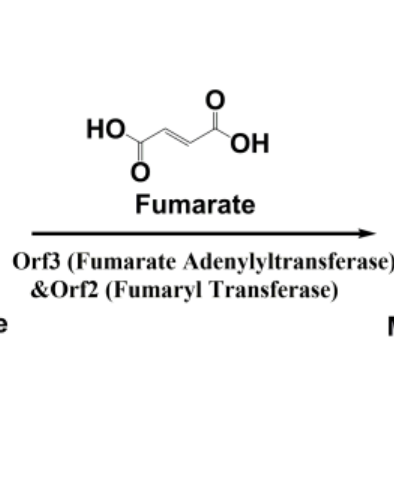

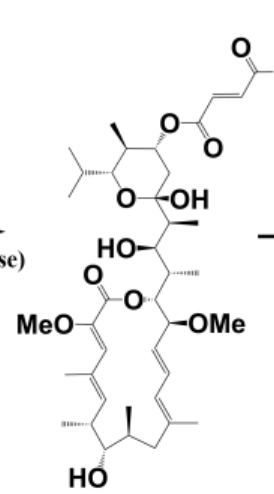

Bafilomycin $\mathrm{C}_{1}$

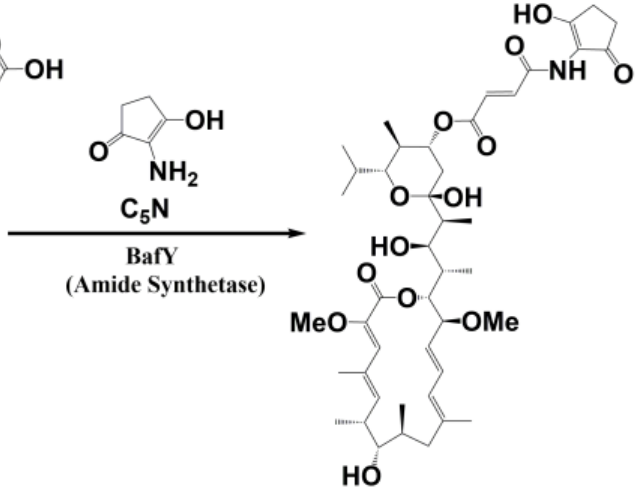

Bafilomycin $\mathrm{B}_{1}$

Figure 2. (a) The bafilomycin biosynthetic gene cluster of S. lohii. (bafAI-AV: PKS genes; bafH: thioesterase gene; bafX-Z and orf 2-3: post-PKS tailoring genes; bafG and orf1: regulatory genes; bafB-bafF: the genes for methoxymalonyl-CoA biosynthesis); (b) The bafilomycin post-PKS tailoring steps.

Except for those characterized as biosynthetic enzymes, bafG and orf1 are the only two rest genes in the baf gene cluster with unclear functionality. According to the previous bioinformatics analysis [20], bafG and orf1 likely encode an AfsR family transcriptional regulator and a LuxR family transcriptional regulator, respectively. To construct bafilomycin high-producers, however, it is necessary to understand the regulatory roles of $b a f G$ and orf1 in the production of bafilomycins. In this work, we first optimized the fermentation medium for bafilomycin production. Subsequently, the regulatory roles of orf1 and bafG in bafilomycin biosynthesis were characterized through gene inactivation and overexpression. By knocking out orf 2 and orf 3 that are responsible for the conversion of bafilomycin $\mathrm{A}_{1}$ to $\mathrm{C}_{1}$, together with orf1 overexpression, a bafilomycin $\mathrm{A}_{1}$ high-producing strain (SLO08) with the titer of $535.1 \pm 25.0 \mathrm{mg} / \mathrm{L}$ in shaking flasks was successfully engineered. We anticipate that this strain will be utilized in the future to produce bafilomycin $A_{1}$ in a cost-effective and eco-friendly manner for pharmacological researches and new drug development efforts.

\section{Results}

\subsection{Optimization of the Fermentation Medium}

We elected to optimize the fermentation medium since the accumulative production of bafilomycins $\mathrm{A}_{1}, \mathrm{~B}_{1}$, and $\mathrm{C}_{1}$ by the wild type $S$. lohii strain (SLO-01, Table 1) was low $(<40 \mathrm{mg} / \mathrm{L})$ upon a 7-day fermentation. According to the previous study [24], soybean oil was considered as a cheap carbon source for Streptomyces to efficiently generate acyl-CoAs, which are common precursors of polyketide natural products. To test if soybean oil can also boost the production of bafilomycins, the fermentation media with different concentrations $(w / v)$ of soybean oil $(0,3 \%, 6 \%, 9 \%$, and $12 \%)$ were used to culture the wild type S. lohii strain. As shown in Figure 3, the bafilomycin production was significantly increased by addition of soybean oil. Specifically, $6 \%$ soybean oil resulted in the highest production of bafilomycins, corresponding to a 5.3-fold enhancement in bafilomycins production by S. lohii when compared to that in the same fermentation medium without soybean oil. Thus, the $6 \%$ soybean oil containing broth was used as the optimized fermentation medium for the production of bafilomycins in the following experiments. 
Table 1. Bacterial strains and plasmids.

\begin{tabular}{ccc}
\hline Strain or Plasmid & Characteristics & Reference \\
\hline Escherichia coli strains & Cloning host & \\
DH5a & Interspecies conjugation & {$[25]$} \\
ET12567/pUZ8002 & Streptomyces lohii ATCC BAA-1276 & {$[26]$} \\
Streptomyces strains & (wild-type strain) & {$[20]$} \\
SLO-01 & S. lohii $\Delta$ bafG & This study \\
SLO-02 & S. lohii $\Delta$ orf1 & This study \\
SLO-03 & S. lohii/pSET152-ermE*-bafG & This study \\
SLO-04 & S. lohii/pSET152-ermE*-orf1 & This study \\
SLO-05 & S. lohii/pSET152-ermE & This study \\
SLO-06 & S. lohii $\Delta$ orf2Eorf3 & {$[9]$} \\
SLO-07 & A. lohii $\Delta$ orf2Eorf3/pSET152s-ermE*-orf1 & This study \\
SLO-08 & Apramycin resistance & {$[27]$} \\
Plasmids & Spectinomycin resistance & This study \\
pSET152-ermE* & Spectinomycin resistance & {$[28]$} \\
pSET152s-ermE* & Ampicillin and Apramycin resistance & {$[29]$} \\
pIJ778 & &
\end{tabular}

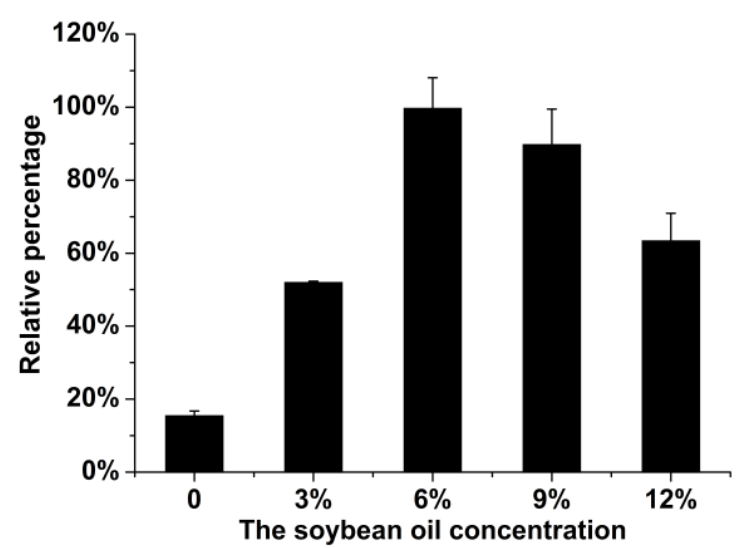

Figure 3. The relative percentage of bafilomycin production by wild-type $S$. lohii when supplied with different concentrations $(w / v)$ of soybean oil in fermentation media. Note: The average bafilomycin production in the fermentation medium with $6 \%$ soybean oil is assigned as $100 \%$.

\subsection{Bioinformatics Analysis of the Regulatory Genes bafG and orf1}

The proteins encoded by bafG (BafG, 609 amino acids) and orf1 (Orf1, 117 amino acids) show high sequence similarity with the AfsR family regulatory proteins and the LuxR family regulatory proteins from different Streptomyces spp., respectively (Figures S1 and S2). To investigate the evolutionary relationship between BafG and the select number of AfsR family regulators from Streptomyces, the phylogenetic tree was built based on their amino acid sequences using the neighbor-joining method [30] (Figure 4a). This phylogenetic analysis confirmed that BafG is indeed an AfsR family member (Figure 4a). According to multiple sequence alignment (Figure S1) and BLAST analysis, BafG exhibits several typical conservative DNA binding domains including the transcriptional regulatory protein, $C$ terminal domain (trans_reg_C domain: 6-73 aa), the bacterial transcriptional activation domain (BTA domain: 81-225 aa), and the nucleotide-binding adaptor shared by APAF-1, R proteins, and CED-4 domain (NB-ARC domain: 302-479 aa); three 34-aa tetratricopeptide repeat motifs (TPR motifs: 81-114 aa, 136-169 aa, and 173-206 aa), which is responsible for recruiting RNA polymerase to bind to the promoter of target genes [31]; and several key residues related to the nucleotides recognition: ${ }^{24} \mathrm{SerVal}^{25}$, Thr44, Thr47, ${ }^{55} \mathrm{SerLeu}^{56}$, Thr66, and Gly70. Compared with the 993-aa AfsR, the C-terminus of 609-aa BafG lacks four TPR repeats which has been validated to be dispensable for the basic function of AfsR 
as a transcriptional activator [31]. Furthermore, there is a unique "TTA" (Leu173) in the coding sequence of bafG, which is the rarest codon in the high-GC-content Streptomyces genomes (Figure S3) [32,33]. This rare codon strongly suggests the possibility that bafG might participate in the regulation of bafilomycin biosynthesis since the involvement of the genes with a "TTA" codon in regulating cell differentiation and antibiotics production has been proposed for other Streptomyces species [32].

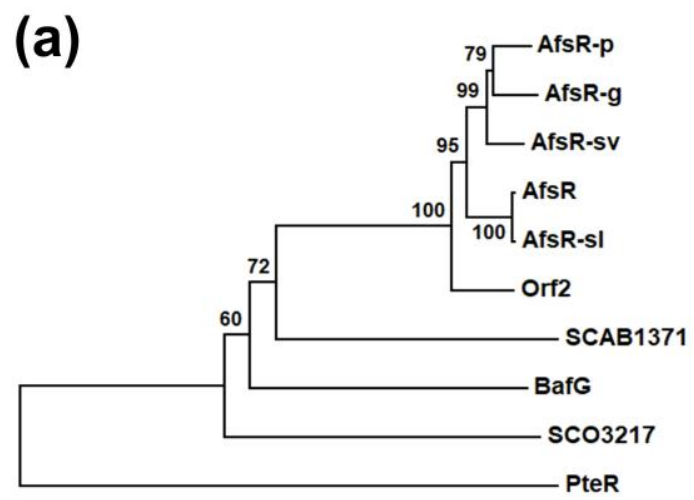

$\stackrel{\longmapsto}{~}$

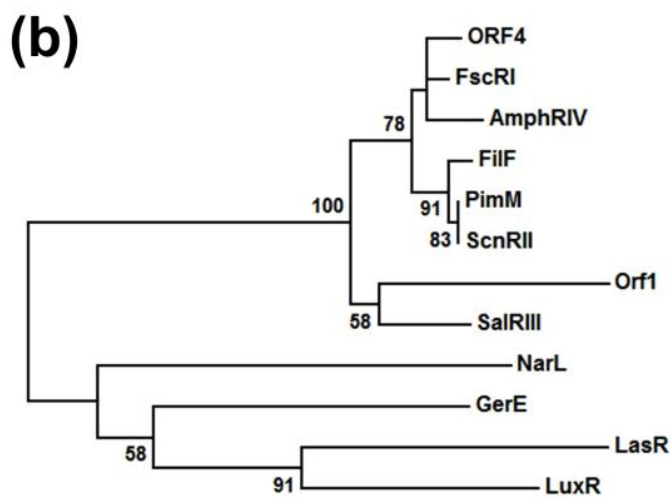

$\underset{0.05}{\longmapsto}$

Figure 4. The neighbor-joining trees of (a) BafG with other identified Streptomyces AfsR homologues and (b) Orf1 with other identified LuxR family proteins. AfsR family proteins: AfsR (993 aa, GenBank accession number: BAA14186.1) and SCO3217 (638 aa, NP_627431.1) from Streptomyces coelicolor A3(2), AfsR-sl from Streptomyces lividans TK 24 (993 aa, EFD67634.1), AfsR-sv from Streptomyces venezuelae ATCC 15439 (1056 aa, ABR08660.1), SCAB1371 from Streptomyces scabies 87.22 (641 aa, CBG67361.1), AfsR-p from Streptomyces peucetius ATCC 27952 (982 aa, CAH10136.1), PteR from Streptomyces avermitilis MA4680 (1096 aa, NP_821585.1), AfsR-g from Streptomyces griseus (974 aa, BAA83790.1), and Orf2 from Streptomyces acidiscabies ATCC 49003 (990 aa, BAO31545.1); LuxR family proteins: LuxR from Vibrio fischeri ATCC 7744 (250 aa, CAA68561.1), SalRIII from Streptomyces albus (231 aa, ABG02265.1), ORF4 from Streptomyces noursei ATCC 11455 (210 aa, AAF71781.1), FscRI from Streptomyces sp. FR-008 (222 aa, AAQ82551.1), PimM from Streptomyces natalensis (192 aa, CAM35468.1), ScnRII from Streptomyces chattanoogensis (192 aa, ADX66474.1), AmphRIV from Streptomyces nodosus (243 aa, WP_079161981.1), FilF from Streptomyces filipinensis (192 aa, AKX77828.1), LasR from Pseudomonas aeruginosa (239 aa, BAA06489.1), GerE from Bacillus subtilis (74 aa, CAA11701.1), and NarL from Escherichia coli K-12 (216 aa, AKK17394.1). The bars show the distance representing 0.1 (a) and 0.05 (b) substitutions per amino acid position and the bootstrap percentages under $50 \%$ from 1000 replicates are hidden.

With respect to Orf1, this small protein displays $>45 \% / 68 \%$ identity/similarity to several identified LuxR family members from Streptomyces (Figure S2). A phylogenetic tree was built using a number of LuxR family proteins including LuxR from Vibrio fischeri ATCC 7744 [34], GerE from Bacillus subtilis [35], LasR from Pseudomonas aeruginosa [36], NarL from Escherichia coli K-12 [37], and so on (Figure 4b). The phylogenetic analysis clearly indicated Orf1 is a LuxR family transcriptional regulator. Specifically, the protein sequence alignment result showed that there is a highly conserved helix-turn-helix (HTH) motif at the C-terminus of Orf1 (41-95 aa), which is a common feature of LuxR family regulators (Figure S2) [38]. At the $N$-termini of many LuxR family members, there generally exist a receptor for inducer binding [39], such as the Per-Arnt-Sim (PAS) domains of SalRIII [40], PimM [41], and FscRI [42]. However, Orf1 only has a C-terminal HTH motif as GerE, suggesting that Orf1 might be an inducer-independent LuxR homologue capable of directly activating the transcription of related genes as the $N$-truncated LuxR $(\Delta 2-162)$ [43].

\subsection{The Regulatory Roles of bafG and orf1 in Bafilomycin Production}

To probe the functions of the afs $R$ family member $b a f G$ and the $l u x R$ family regulatory gene orf1 for bafilomycin production in S. lohii, a 682-bp internal fragment of bafG or a 335-bp internal fragment of orf1 was in-frame replaced by the aac(IV) cassette via 
homologous recombination, giving rise to the bafG deletion mutant SLO-02 and the orf1 deletion mutant SLO-03, respectively (Figure S4). As results, SLO-02 and SLO-03 without any cell growth and morphological difference completely lost the ability to produce any bafilomycins (Figure 5). These results suggested that the disruption of bafG or orf 1 might transcriptionally inactivate/repress some key biosynthetic genes in the baf cluster. Next, bafG and orf1 were overexpressed in S. lohii by generating the strains SLO-04 (S. lohii/pSET152-ermE*-bafG, Table 1) and SLO-05 (S. lohii/pSET152-ermE*-orf1, Table 1). Compared with the wild type S. lohii, the transcriptional levels of orf1 and bafG in these two mutants, without any cell growth difference observed, were significantly increased at $12 \mathrm{~h}$ and $36 \mathrm{~h}$ under the strong promoter $\mathrm{ermE}^{*}$ (Figure 6). Furthermore, wild-type S. lohii produced $220.3 \pm 10.9 \mathrm{mg} / \mathrm{L}$ of bafilomycins in the optimized fermentation medium within 7 days in the $250 \mathrm{~mL}$ shaking flasks (Figures 7 and 8), while the $b a f G$ overexpression strain SLO-04 and the orf1 overexpression strain SLO-05 generated $338.9 \pm 10.7 \mathrm{mg} / \mathrm{L}$ and $508.5 \pm 41.2 \mathrm{mg} / \mathrm{L}$ of bafilomycins in parallel fermentations, respectively, corresponding to 1.5- and 2.3-fold higher total bafilomycins titers than the wild type strain (Figures 7 and 8). The bafilomycin $\mathrm{A}_{1}$ titers of SLO-04 (271.1 $\left.\pm 19.7 \mathrm{mg} / \mathrm{L}\right)$ and SLO-05 $(423.5 \pm 29.5 \mathrm{mg} / \mathrm{L})$ increased 0.8- and 1.8-fold relative to that of wild-type S. lohii (152.9 $\pm 11.4 \mathrm{mg} / \mathrm{L})$, respectively (Figures 7 and 8). These titer improvements could be qualitatively rationalized by the quantitative real-time PCR (qRT-PCR) results (Figure 6): the overexpression of orf1 in SLO-05 led to a 4.9- and 1.4-fold improvement of the transcriptional level of bafAV, the final PKS gene for bafilomycin biosynthesis, when compared with wild-type $S$. lohii at $12 \mathrm{~h}$ and $36 \mathrm{~h}$, respectively; and the transcriptional level of bafAV in SLO-04 was increased by 4.7- and 0.7-fold compared to that of wild-type S. lohii at $12 \mathrm{~h}$ and $36 \mathrm{~h}$, respectively. These results strongly suggested that $b a f G$ and orf1 are indeed positive transcriptional factors for bafilomycin biosynthesis in S. lohii.

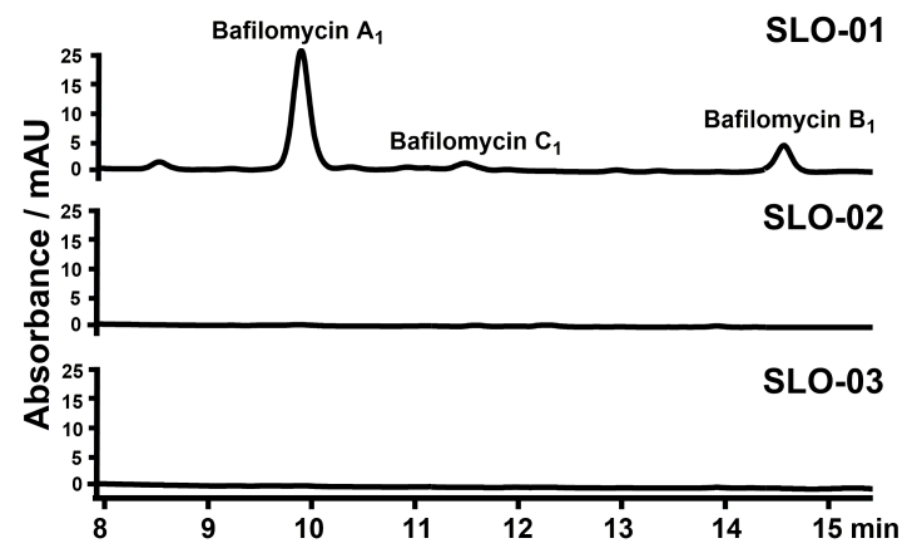

Figure 5. HPLC analysis (254 nm) of the fermentation broth of wild-type S. lohii and the bafG/orf1 inactivation strains.

(a)

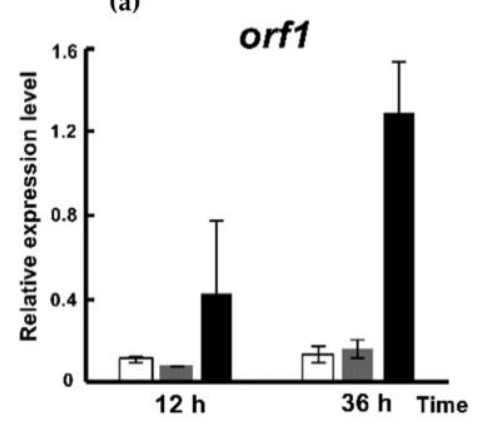

(b)

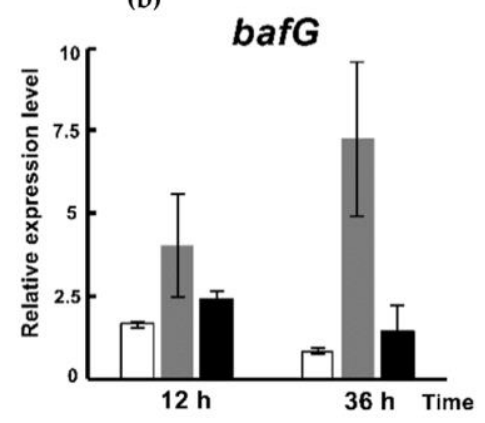

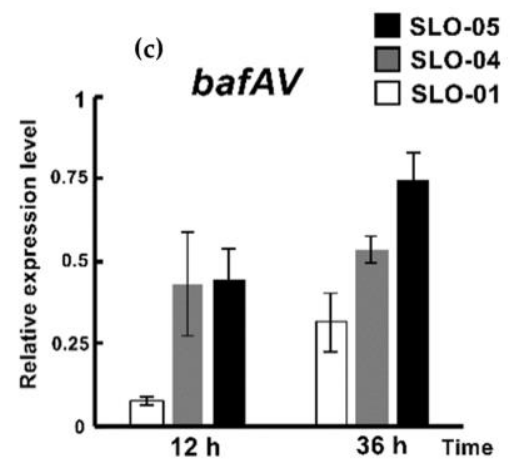

Figure 6. The relative transcriptional levels of (a) orf1, (b) bafG, and (c) bafAV in wild-type and the orf1/bafG overexpressed S. lohii strains at $12 \mathrm{~h}$ and $36 \mathrm{~h}$. 


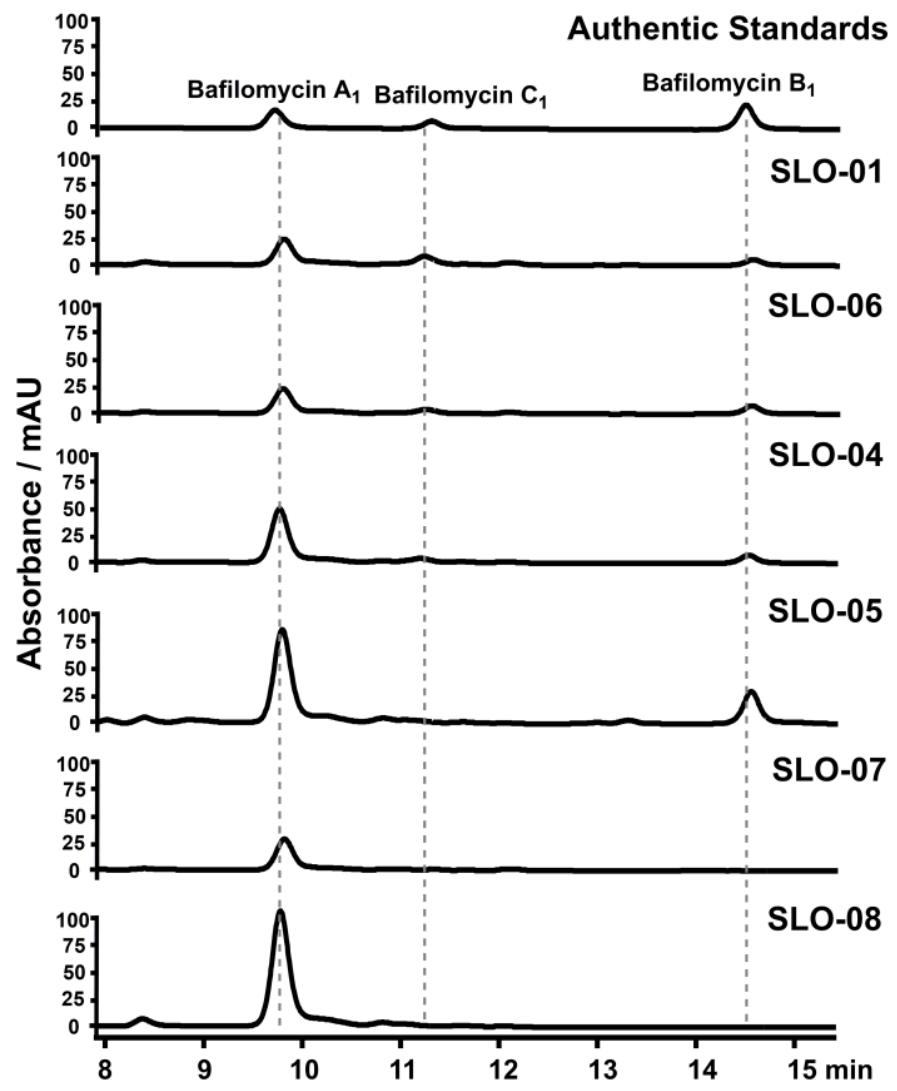

Figure 7. HPLC analysis $(254 \mathrm{~nm})$ of the bafilomycin production by wild-type and mutant S. lohii strains.

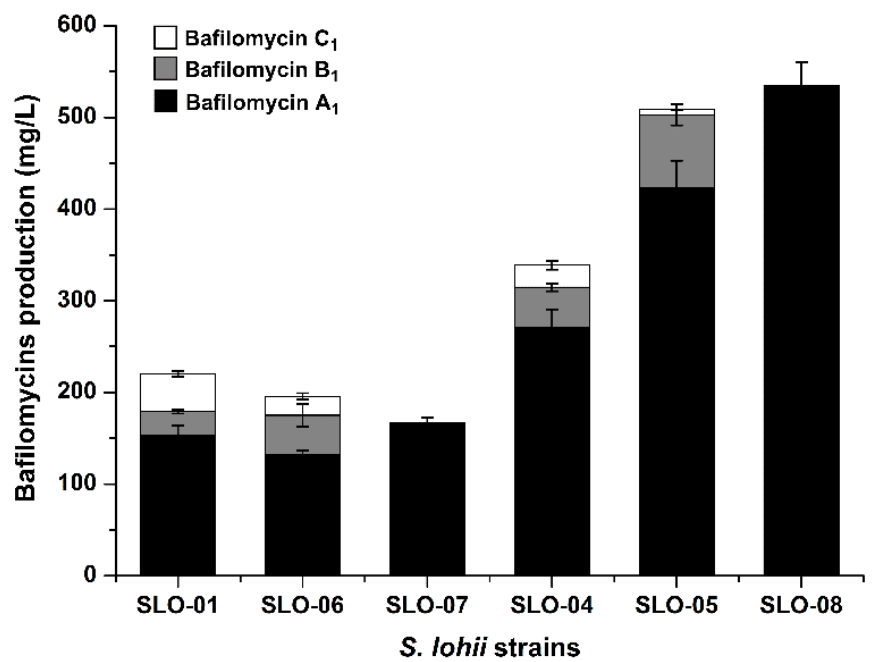

Figure 8. The bafilomycin production (mg/L) by wild-type and mutant S. lohii strains.

\subsection{Construction of Bafilomycin $A_{1}$ High-Producing Strains}

In our previous study [9], SLO-07 (S. lohii $\Delta$ orf2Eorf3, Table 1), in which the region encoding orf2 and orf3 was replaced by aac(IV) to disrupt the bafilomycin post-PKS tailoring steps in S. lohii, was constructed. In this study, this strain solely produced bafilomycin $\mathrm{A}_{1}$ $(167.3 \pm 5.4 \mathrm{mg} / \mathrm{mL})$ under the optimized fermentation conditions (Figures 7 and 8). To further construct a bafilomycin $\mathrm{A}_{1}$ high-producing strain, orf1, whose overexpression led to higher bafilomycin $A_{1}$ production than that of $b a f G$, was chosen to be introduced into SLO-07, giving rise to SLO-08 (Table 1). Upon overexpression of orf1 in the strain that purely produces bafilomycin $A_{1}$, the resultant yield of bafilomycin $A_{1}$ reached $535.1 \pm 25.0 \mathrm{mg} / \mathrm{L}$ (Figures 7 and 8), representing the highest reported bafilomycin $A_{1}$ production to date. 


\section{Discussion}

Generally, oil is one of excellent carbon sources commonly used in the fermentation media of Streptomyces to support cell growth and metabolites [24]. More importantly, soybean oil has been used as a low-cost feedstock to enhance the supply of biosynthetic precursors for improvement of polyketide production in Streptomyces since fatty acids can be directly bioconverted into acyl-CoAs as the precursors of polyketides [24,44,45]. For example, the FK506 production in Streptomyces tsukubaensis was increased 0.9-fold by feeding soybean oil into the production medium [24]. Here, the initial fermentation medium for S. lohii was optimized through investigating the relationship between soybean oil concentrations and bafilomycin production; and 6\% soybean oil showed the best improving effect. Based on our analysis of the draft genome of S. lohii, at least forty esterase/lipase genes were revealed, suggesting that soybean oil could be efficiently utilized via primary metabolism for both cell growth (the biomass of $S$. lohii was significantly enhanced in the optimized medium) and bafilomycin production. In the optimized fermentation medium, the dominant product of the wild type $S$. lohii was bafilomycin $\mathrm{A}_{1}$, accounting for about $70 \%$ of total production of bafilomycins, although bafilomycin $B_{1}$ is the primary product when using the MD2 medium for S. lohii fermentation in our previous study [20]. Thus, using different fermentation materials to regulate the bafilomycin production and product distribution will be our next goal.

In this work, BafG and Orf1 were identified as the positive AfsR family regulator and the positive LuxR family regulator, respectively. AfsR was firstly characterized by Horinouchi et al. as a global activator involved in the regulatory cascades and antibiotics production in the type strain Streptomyces coelicolor A3(2) [46,47]. The activity of AfsR for enhancing the transcription of $a f_{s} S$ can be significantly improved after the phosphorylation of its threonine and serine residues by AfsK [47]. Generally, AfsR family proteins work as transcriptional activators in secondary metabolite biosynthesis. For example, the overexpression of afsR-sv in S. venezuelae ATCC 15439 improved the production of pikromycin [48]; SCAB1371 was identified as a positive transcriptional regulator for pyochelin biosynthesis in the plant pathogen Streptomyces scabies 87-22 [49]; the overexpression of afsR in Streptomyces lomondensis led to up-regulation of two genes related to lomofungin biosynthesis and increased the lomofungin production by 2.5 -fold [50]. Similarly, the disruption of the bafG homologue bfmH in Kitasatospora setae KM-6054 also led to the abolishment of bafilomycins [8]. The characterization of $b a f G$ will expand the pool of the afs $R$ family regulatory genes and it may be overexpressed in other Streptomyces species for isolation of novel compounds through activating silent genes or for improvement of the target antibiotics production, which is currently ongoing in our laboratory. However, there is no any afs $K$ homologue in the baf cluster, which suggests that BafG might be phosphorylated by an AfsK homologue outside the gene cluster.

LuxR was firstly characterized in the lux operon of Vibrio fischeri ATCC 7744, which is a cell density-dependent transcriptional activator involved in luciferase biosynthesis and play important roles in acyl-homoserine lactones-mediated quorum sensing [34,39]. To date, hundreds of LuxR family members have been discovered by genome mining and bioinformatics predictions. Of note, dozens of LuxR family regulatory factors have been proved to participate in Streptomyces secondary metabolite biosynthesis, such as PikD, which was identified as a pathway-specific positive regulator for pikromycin biosynthesis in Streptomyces venezuelae [51]; TmcN, the activator of tautomycetin biosynthesis in Streptomyces sp. CK4412 [52]; and $\mathrm{RapH}$, a putative transcriptional regulator for rapamycin biosynthesis in Streptomyces hygroscopicus [53]. Generally, the N-terminal motifs of LuxR family proteins are bound by the quorum-sensing molecules to relieve the repression by the $C$-terminal motif responsible for activating the transcription of related genes [39]. In this study, the results of orf 1 inactivation and overexpression strongly suggest its positive regulatory role in bafilomycin biosynthesis of $S$. lohii. To our surprise, Orf1 was identified as a LuxR family protein with only a conservative $C$-terminal HTH motif, suggesting it 
may function as an inducer-independent activator, similar to GerE and the $N$-truncated $\operatorname{LuxR}(\Delta 2-162)[35,43]$.

In previous studies, the gamma-butyrolactone synthetase/autoregulator receptor homologues were found to play vital roles in bafilomycin production. Specifically, the deletion of the gamma-butyrolactone synthetase gene homologue stcA from Streptomyces sp. SBI034 led to the complete abolishment of bafilomycin production as well as aerial mycelium formation and sporulation [54]; the deletion of gamma-butyrolactone autoregulator receptor genes $k s b A$ and $k s b C$ in Kitasatospora setae indicated that KsbA and KsbC respectively control bafilomycin production and aerial mycelium formation negatively and positively $[55,56]$. However, the regulatory factors involved in bafilomycin biosynthesis has not caught much attention in Streptomyces species. Our study provides some initial understandings of the regulatory roles of the afs $R$ and $\operatorname{lux} R$ family genes in governing the biosynthesis of bafilomycins, which also provide an effective strategy for engineering high bafilomycin producers. Since total synthesis of bafilomycin $A_{1}$ has been proved to be complex and low-yield $[18,19]$, the construction of bafilomycin $A_{1}$ high-producing strains holds great potential of application.

\section{Materials and Methods}

\subsection{Materials}

The chemicals and antibiotics in this study were purchased from Solarbio (Beijing, China) and Sinopharm Chemical Reagent (Beijing, China) unless otherwise specified. Apramycin sulfate was bought from Sangon (Shanghai, China). T4 DNA ligase and all fast-digest restriction endonucleases were bought from Thermo Fisher Scientific (Waltham, MA, USA). I-5 ${ }^{\text {TM }} 2 \times$ High-Fidelity Master Mix obtained from TsingKe (Beijing, China) was used for PCR amplification. ClonExpress Ultra One Step Cloning Kit was purchased from Vazyme (Nanjing, China). MonPure ${ }^{\mathrm{TM}}$ Gel \& PCR Clean Kit and Plasmid Miniprep Kit were bought from Baisai Biotechnology (Qingdao, China). GelRed for agarose gel electrophoresis was purchased from the MDBio (Xinbei, China). The MiniBEST Universal RNA Extraction Kit for RNA extraction and genomic DNA digestion, the PrimeScript ${ }^{\mathrm{TM}}$ RT reagent for cDNA preparation and the TB Green ${ }^{\circledR}$ Premix Ex Taq ${ }^{\mathrm{TM}}$ II (Tli RNaseH Plus) for qRT-PCR were purchased from Takara (Dalian, China).

\subsection{Strains, Plasmids, and Bacterial Growth Conditions}

Strains and plasmids used in this study are listed in Table 1. E. coli DH5a [25] was used as the host strain for plasmid construction, replication, and preservation. E. coli ET12567/pUZ8002 [26] was employed for interspecies conjugation between E. coli and S. lohii. All E. coli strains were cultivated in Luria-Bertani medium ( $10 \mathrm{~g}$ tryptone, $5 \mathrm{~g}$ yeast extract, and $10 \mathrm{~g} \mathrm{NaCl}$ per liter) at $37^{\circ} \mathrm{C}$. The wild type and mutant $S$. lohii strains were grown on MS agar (mannitol $20 \mathrm{~g}$, soybean flour $20 \mathrm{~g}$, and agar $20 \mathrm{~g}$ per liter) at $28^{\circ} \mathrm{C}$ for sporulation and conjugation. $2 \times$ YT liquid medium (16 g tryptone, $10 \mathrm{~g}$ yeast extract, and $5 \mathrm{~g} \mathrm{NaCl}$ per liter) was used to grow $S$. lohii cells for genomic DNA (gDNA) preparation. The initial fermentation medium ( $\mathrm{pH}=7.1$ ) contained $20 \mathrm{~g}$ glucose, $20 \mathrm{~g}$ soybean flour, $2 \mathrm{~g}$ NZ-amine, $1.5 \mathrm{~g}$ corn syrup, $1 \mathrm{~g}$ yeast extract, $8 \mathrm{~g} \mathrm{NaNO}_{3}, 8 \mathrm{~g} \mathrm{CaCO}_{3}, 6 \mathrm{~g}\left(\mathrm{NH}_{4}\right)_{2} \mathrm{SO}_{4}, 5 \mathrm{~g}$ $\mathrm{NaCl}$, and $0.3 \mathrm{~g} \mathrm{~K}_{2} \mathrm{HPO}_{4}$ per liter. The optimized fermentation medium was the initial fermentation medium supplemented with $6 \%$ soybean oil. The concentrations of antibiotics used in this study were as follows: apramycin $(50 \mu \mathrm{g} / \mathrm{mL})$, spectinomycin $(100 \mu \mathrm{g} / \mathrm{mL})$, kanamycin $(50 \mu \mathrm{g} / \mathrm{mL})$, chloramphenicol $(25 \mu \mathrm{g} / \mathrm{mL})$, and nalidixic acid $(25 \mu \mathrm{g} / \mathrm{mL})$.

\subsection{DNA Sequencing and Bioinformatics Analysis}

DNA sequencing and primer synthesis were performed by TsingKe (Qingdao, China). Gene annotation and the collection of amino acid sequences of AfsR and LuxR family members were carried out using NCBI databases (http:/ / www.ncbi.nlm.nih.gov/). The gene promoters were predicted using Softberry online tools (http://linux1.softberry.com/). The phylogenetic analysis was performed by MEGA version 7.0 (Philadelphia, PA, USA) [57] 
using the neighbor-joining method [30]. DNAman 7.0 (San Ramon, Cal, USA) was used for protein sequence alignments.

\subsection{Construction of the Suicide Knockout Vectors}

The primers for vector construction are listed in Table S1. The suicide vector pCIMt002 was kindly provided by Prof. Yihua Chen at Institute of Microbiology, Chinese Academy of Sciences [29]. To generate the suicide knockout vectors for bafG and orf1 inactivation, the upstream and downstream homologous fragments (approximately $2.0 \mathrm{~kb}$ ) of bafG were amplified from the S. lohii gDNA using the primer pairs of bafG-LA-FP/bafG-LA-RP and bafG-RA-FP/bafG-RA-RP, respectively; and the upstream and downstream homologous fragments (about $2.0 \mathrm{~kb}$ ) of orf1 were amplified from the $S$. lohii gDNA using the primer pairs of orf1-LA-FP/orf1-LA-RP and orf1-RA-FP / orf1-RA-RP, respectively. Subsequently, the homologous fragments were cloned into the NcoI and NheI restriction sites of pCIMt002 to generate pCIMt002- $\triangle b a f G$ and pCIMt002-_orf1 using the ClonExpress Ultra One Step Cloning Kit.

\subsection{Gene Inactivation in S. lohii}

The gene orf1 or bafG in S. lohii was replaced by the apramycin resistance cassette $(a a c(I V))$ following the blue-white screening strategy developed by Chen et al. [29]. The suicide vectors pCIMt002- $\triangle b a f G$ or pCIMt002- $\Delta$ orf1 were transferred into S. lohii via $E$. coli-Streptomyces conjugation [26]. Upon an incubation at $28{ }^{\circ} \mathrm{C}$ for $12 \mathrm{~h}$, each MS agar plate (containing $50 \mathrm{mM} \mathrm{CaCl}_{2}$ and $50 \mathrm{mM} \mathrm{MgCl}_{2}$ ) was overlaid with $1 \mathrm{~mL}$ sterilized water containing $1.25 \mathrm{mg}$ apramycin and $0.5 \mathrm{mg}$ nalidixic acid. After a further $28^{\circ} \mathrm{C}$ incubation for 3-5 days, the white colonies indicative of the desired double-crossover recombinants were picked up from the blue colonies indicative of the undesired single-crossover mutants. The genotypes of the two picked mutants (SLO-02 for $\Delta b a f G$ and SLO-03 for $\Delta$ orf1, Table 1) were confirmed by PCR (Figure S4).

\subsection{Construction of Integrative Plasmids for Regulatory Gene Overexpression}

The coding sequences of bafG (1830 bp) and orf1 (354 bp) were amplified using the S. lohii gDNA as template. For bafG, the primer pair was BafG-BamHI-FP/BafG-KpnI$\mathrm{RP}$; and for orf1, the primer pair was Orf1-BamHI-FP/Orf1-KpnI-RP (Table S1). The bafG and orf1 fragments were inserted into the BamHI restriction site of pSET152-ermE ${ }^{*}$ (Bierman et al., 1992) to generate the regulatory gene overexpression vectors pSET152erm $E^{*}$-bafG and pSET152-erm $E^{*}$-orf1, respectively. Since the apramycin resistance gene has already been integrated into the genome of $S$. lohii $\Delta$ orf2Eorf3, the spectinomycin resistance gene ( $a a d A)$ fragment was PCR amplified from pIJ778 (Gust et al., 2003) using the primers Spec-NdeI-FP/Spec-SacI-RP as the second selection marker. Next, the aadA cassette was in-fusion cloned into the SacI-pre-digested pSET152-erm $E^{*}$, yielding the integrative vector pSET152s-ermE* ${ }^{*}$ The coding sequence of orf1 was cloned into pSET152s-erm $E^{*}$ to afford pSET152s-ermE*-orf1 for gene overexpression in S. lohii $\Delta$ orf2Eorf3.

\subsection{Overexpression of Regulatory Genes}

For gene overexpression of bafG or orf1, the integrative plasmid pSET152-erm $E^{*}$-bafG or pSET152-ermE*-orf1 was introduced into wild-type $S$. lohii, respectively, by interspecies conjugation from E. coli ET12567/pUZ8002 [26]. Upon an incubation at $28{ }^{\circ} \mathrm{C}$ for $12 \mathrm{~h}$, each plate was overlaid with $1 \mathrm{~mL}$ sterilized water containing $1.25 \mathrm{mg}$ apramycin and $0.5 \mathrm{mg}$ nalidixic acid. After additional 3-5 days, the recombinants were inoculated onto MS plates with $25 \mu \mathrm{g} / \mathrm{mL}$ nalidixic acid and $50 \mu \mathrm{g} / \mathrm{mL}$ apramycin. The resultant two apramycin resistant strains SLO-04 (S. lohii/pSET152-ermE*-bafG, Table 1) and SLO-05 (S. lohii/pSET152-ermE*-orf1, Table 1) were PCR confirmed using their gDNA as template. 


\subsection{Genotypic Confirmation of S. lohii Mutants}

The primers for PCR confirmation of S. lohii mutants are listed in Table S2. The primers $b a f G-K O-F P / b a f G-K O-R P$ were used for screening the $\triangle b a f G$ mutants. The expected length of the PCR fragments from the wild type and the $\triangle b a f G$ mutants is $916 \mathrm{bp}$ and $1227 \mathrm{bp}$, respectively (Figure S4a,b). The primers orf1-KO-FP/orf1-KO-RP were used for screening the $\Delta$ orf1 mutants. The expected length of the PCR fragments from the wild type and the $\Delta$ orf 1 mutants is $562 \mathrm{bp}$ and $1220 \mathrm{bp}$, respectively (Figure S4c,d). The primers M13F47/M13R-48 were used to screen the orf1 and bafG overexpression strains (Figure S5): the expected length of the PCR product from SLO-06 (Table 1) is $471 \mathrm{bp}$; the expected length of PCR products of SLO-05 and SLO-08 (Table 1) is $831 \mathrm{bp}$; and the expected length of $\mathrm{PCR}$ products of SLO-04 is $2307 \mathrm{bp}$. All the PCR fragments were gel purified and further confirmed by DNA sequencing.

\subsection{Fermentation and HPLC Analysis}

A single colony of the wild type or each mutant of $S$. lohii was used to inoculate $30 \mathrm{~mL}$ $2 \times$ YT medium, and cultured at $220 \mathrm{rpm}, 28^{\circ} \mathrm{C}$. After 2 days, $3 \mathrm{~mL}$ seed culture was inoculated into $30 \mathrm{~mL}$ fermentation medium and cultivated at $28^{\circ} \mathrm{C}, 250 \mathrm{rpm}$ for another 7 days. Next, $200 \mu \mathrm{L}$ fermentation culture was extracted by adding $600 \mu \mathrm{L}$ methanol, vortexed for $30 \mathrm{~min}$, and centrifuged at $14,000 \times \mathrm{g}$ for $10 \mathrm{~min}$. The supernatants were directly used for reverse phase HPLC analysis $(254 \mathrm{~nm})$ with a Thermo C-18 column $(4.6 \times$ $150 \mathrm{~mm}$ ) under a liner gradient of $60-100 \%$ acetonitrile over $15 \mathrm{~min}, 100 \%$ acetonitrile for $5 \mathrm{~min}$, and $100-60 \%$ acetonitrile over $2 \mathrm{~min}$ in deionized $\mathrm{H}_{2} \mathrm{O}$ (with $0.1 \%$ trifluoroacetic acid) at a flow rate of $1 \mathrm{~mL} / \mathrm{min}$. The fermentation of the wild type and all mutant $S$. lohii strains were carried out in duplicate, and the production of bafilomycins was quantified based on the integrated peak areas using authentic bafilomycin $A_{1}, B_{1}$, and $C_{1}$ as standards [9].

\subsection{Transcriptional Analysis of the Wild Type and Mutant S. lohii Strains by qRT-PCR}

The mycelia of the wild type or mutant $S$. lohii strains in the optimized fermentation media were collected at 12 and $36 \mathrm{~h}$. The total RNA was extracted with the genomic DNA removed using the MiniBEST Universal RNA Extraction Kit, and was reversely transcribed using random primer mix by following the product manual. The primers for qRT-PCR were designed by Primer3Plus online service (http:/ / www.primer3plus.com/cgi-bin/dev/ primer3plus.cgi) and listed in Table S3. The transcription of target genes was determined by qRT-PCR on a LightCycler 480 II (Roche Life Science, Basel, Switzerland) in triplicate. For determination of the relative transcription levels, the data were normalized to the housekeeping gene $h r d B$ in S. lohii and quantified by the $2^{-\Delta \Delta C T}$ method [58].

Supplementary Materials: The following are available online at https:/ / www.mdpi.com/1660-339 7/19/1/29/s1, Figure S1: Multiple protein sequence alignment of BafG with several AfsR family proteins, Figure S2: Multiple protein sequence alignment of Orf1 with several LuxR family proteins, Figure S3: The partial open reading frame of BafG (141-210 aa), Figure S4: Inactivation of bafG and orf1, Figure S5: The overexpression of bafG/orf1, Table S1: The primers for construction of knock-out and regulatory genes overexpression vectors, Table S2: The primers for construction of knock-out vectors and PCR confirmation of $S$. lohii mutants, Table S3: The primers for quantitative real-time PCR.

Author Contributions: Conceptualization, Z.L. and S.L. (Shengying Li); methodology, Z.L., L.D., X.Z., and W.L.; software, Z.L., L.D., and Y.J.; validation, Z.L. and S.L. (Shuai Li); formal analysis, Z.L., S.L. (Shuai Li) and Y.J.; investigation, Z.L.; data curation, Z.L. and S.L. (Shengying Li); writing-original draft preparation, Z.L.; writing — review and editing, Z.L. and S.L. (Shengying Li); visualization, Z.L.; supervision, S.L. (Shengying Li); project administration, S.L. (Shengying Li); funding acquisition, S.L. (Shengying Li) and W.Z. All authors have read and agreed to the published version of the manuscript.

Funding: This work was funded by the National Key Research and Development Program of China (2019YFA0905704 and 2019YFA0905100), the National Natural Science Foundation of China (32025001, 82022066, 81741155, and 32000039), Weihai Science and Technology Development Program 
(2018ZC11), Shandong Provincial Natural Science Foundation (ZR2019ZD20), the Laboratory for Marine Drugs and Bioproducts of Pilot National Laboratory for Marine Science and Technology (Qingdao) (LMDBKF-2019-01), and the State Key Laboratory of Bio-organic and Natural Products Chemistry (SKLBNPC18242).

Institutional Review Board Statement: Not applicable.

Informed Consent Statement: Not applicable.

Data Availability Statement: Data is contained within the article or supplementary materials.

Acknowledgments: We are grateful to Yihua Chen and Pengwei Li at Institute of Microbiology, Chinese Academy of Sciences for providing us with the plasmids and protocols for the efficient blue-white screening based gene inactivation system for Streptomyces.

Conflicts of Interest: The authors declare no conflict of interest.

\section{References}

1. Werner, G.; Hagenmaier, H.; Dpautz, H.; Baumgartner, A.; Zähner, H. Metabolic products of microorganisms. 224. Bafilomycins, a new group of macrolide antibiotics. Production, isolation, chemical structure and biological activity. J. Antibiot. 1984, 37, 110-117. [CrossRef]

2. Kinashi, H.; Someno, K.; Sakaguchi, K. Isolation and characterization of concanamycins A, B and C. J. Antibiot. 1984, 37, 1333-1343. [CrossRef] [PubMed]

3. Wilton, J.; Hokanson, G.; French, J.C. PD 118,576: A new antitumor macrolide antibiotic. J. Antibiot. 1985, 38, 1449-1452. [CrossRef] [PubMed]

4. Bowman, E.J.; Siebers, A.; Altendorf, K. Bafilomycins: A class of inhibitors of membrane ATPases from microorganisms, animal cells, and plant cells. Proc. Natl. Acad. Sci. USA 1988, 85, 7972-7976. [CrossRef] [PubMed]

5. Werner, G.; Hagenmaier, H.; Albert, K.; Kohlshorn, H. The structure of the bafilomycins, a new group of macrolide antibiotics. Tetrahedron Lett. 1983, 24, 5193-5196. [CrossRef]

6. Chen, Y.; Lu, M.; Chung, H.; Weng, C.; Su, J.; Yang, Y.; Su, Y.; Chang, Y.; Kuo, J.; Wu, Y. Bafilomycin M, a new cytotoxic bafilomycin produced by a Streptomyces sp. isolated from a marine sponge Theonella sp. Tetrahedron Lett. 2016, 57, 4863-4865. [CrossRef]

7. Li, J.-Q.; Zhao, H.-W.; Ma, Z.-J. Cytotoxic bafilomycin analogues 6/5/5 with tricyclic ring system from a marine-derived Streptomyces sp. Tetrahedron Lett. 2020, 61, 151874. [CrossRef]

8. Nara, A.; Hashimoto, T.; Komatsu, M.; Nishiyama, M.; Kuzuyama, T.; Ikeda, H. Characterization of bafilomycin biosynthesis in Kitasatospora setae KM-6054 and comparative analysis of gene clusters in Actinomycetales microorganisms. J. Antibiot. 2017, 70, 616-624. [CrossRef]

9. Li, Z.; Du, L.; Zhang, W.; Zhang, X.; Jiang, Y.; Liu, K.; Men, P.; Xu, H.; Fortman, J.L.; Sherman, D.H. Complete elucidation of the late steps of bafilomycin biosynthesis in Streptomyces lohii. J. Biol. Chem. 2017, 292, 7095-7104. [CrossRef]

10. Wu, C.; Medema, M.H.; Läkamp, R.M.; Zhang, L.; Dorrestein, P.C.; Choi, Y.H.; van Wezel, G.P. Leucanicidin and endophenasides result from methyl-rhamnosylation by the same tailoring enzymes in Kitasatospora sp. MBT66. ACS Chem. Biol. 2015, 11, 478-490. [CrossRef]

11. Mauvezin, C.; Nagy, P.; Juhász, G.; Neufeld, T.P. Autophagosome-lysosome fusion is independent of V-ATPase-mediated acidification. Nat. Commun. 2015, 6, 7007. [CrossRef] [PubMed]

12. Del Poeta, M.; Cruz, M.C.; Cardenas, M.E.; Perfect, J.R.; Heitman, J. Synergistic antifungal activities of bafilomycin $A_{1}$, fluconazole, and the pneumocandin MK-0991/caspofungin acetate (L-743,873) with calcineurin inhibitors FK506 and L-685,818 against Cryptococcus neoformans. Antimicrob. Agents Chemother. 2000, 44, 739-746. [CrossRef] [PubMed]

13. Kawaguchi, T.; Miyazawa, K.; Moriya, S.; Ohtomo, T.; Che, X.; Naito, M.; Itoh, M.; Tomoda, A. Combined treatment with bortezomib plus bafilomycin $\mathrm{A}_{1}$ enhances the cytocidal effect and induces endoplasmic reticulum stress in U266 myeloma cells: Crosstalk among proteasome, autophagy-lysosome and ER stress. Int. J. Oncol. 2011, 38, 643.

14. Li, L.Q.; Xie, W.J.; Pan, D.; Chen, H.; Zhang, L. Inhibition of autophagy by bafilomycin $\mathrm{A}_{1}$ promotes chemosensitivity of gastric cancer cells. Tumor Biol. 2016, 37, 653-659. [CrossRef] [PubMed]

15. Yang, N.; Shen, H.M. Targeting the Endocytic Pathway and Autophagy Process as a Novel Therapeutic Strategy in COVID-19. Int. J. Biol. Sci. 2020, 16, 1724-1731. [CrossRef] [PubMed]

16. Dröse, S.; Altendorf, K. Bafilomycins and concanamycins as inhibitors of V-ATPases and P-ATPases. J. Exp. Biol. 1997, $200,1-8$.

17. Gagliardi, S.; Gatti, P.A.; Belfiore, P.; Zocchetti, A.; Clarke, G.D.; Farina, C. Synthesis and structure-activity relationships of bafilomycin $\mathrm{A}_{1}$ derivatives as inhibitors of vacuolar $\mathrm{H}^{+}$-ATPase. J. Med. Chem. 1998, 41, 1883-1893. [CrossRef] [PubMed]

18. Kleinbeck, F.; Carreira, E.M. Total synthesis of bafilomycin A . Angew. Chem. Int. Edit. 2009, 48, 578-581. [CrossRef]

19. Scheidt, K.A.; Bannister, T.D.; Tasaka, A.; Wendt, M.D.; Savall, B.M.; Fegley, G.J.; Roush, W.R. Total synthesis of (-)-bafilomycin A 1 . J. Am. Chem. Soc. 2002, 124, 6981-6990. [CrossRef]

20. Zhang, W.; Fortman, J.L.; Carlson, J.C.; Yan, J.; Liu, Y.; Bai, F.; Guan, W.; Jia, J.; Matainaho, T.; Sherman, D.H.; et al. Characterization of the bafilomycin biosynthetic gene cluster from Streptomyces lohii. ChemBioChem 2013, 14, 301-306. [CrossRef] 
21. Hwang, J.Y.; Kim, H.S.; Kim, S.H.; Oh, H.R.; Nam, D.H. Organization and characterization of a biosynthetic gene cluster for bafilomycin from Streptomyces griseus DSM 2608. AMB Express 2013, 3, 1-9. [CrossRef] [PubMed]

22. Hwang, J.Y.; Kim, S.H.; Oh, H.R.; Kwon, E.; Nam, D.H. Analysis of a draft genome sequence of Kitasatospora cheerisanensis KCTC 2395 producing bafilomycin antibiotics. J. Microbiol. 2015, 53, 84-89. [CrossRef] [PubMed]

23. Yin, M.; Li, G.; Jiang, Y.; Han, L.; Huang, X.; Lu, T.; Jiang, C. The complete genome sequence of Streptomyces albolongus YIM 101047, the producer of novel bafilomycins and odoriferous sesquiterpenoids. J. Biotechnol. 2017, 262, 89-93. [CrossRef] [PubMed]

24. Wang, J.; Liu, H.; Huang, D.; Jin, L.; Wang, C.; Wen, J. Comparative proteomic and metabolomic analysis of Streptomyces tsukubaensis reveals the metabolic mechanism of FK506 overproduction by feeding soybean oil. Appl. Microbiol. Biotechnol. 2017, 101, 2447-2465. [CrossRef] [PubMed]

25. Hanahan, D. Studies on transformation of Escherichia coli with plasmids. J. Mol. Biol. 1983, 166, 557-580. [CrossRef]

26. Kieser, T. Practical Streptomyces Genetics; John Innes Foundation: Norwich, UK, 2000.

27. Bierman, M.I.; Logan, R.; Obrien, K.; Seno, E.T.; Rao, R.N.; Schoner, B.E. Plasmid cloning vectors for the conjugal transfer of DNA from Escherichia coli to Streptomyces spp. Gene 1992, 116, 43-49. [CrossRef]

28. Gust, B.; Challis, G.L.; Fowler, K.; Kieser, T.; Chater, K.F. PCR-targeted Streptomyces gene replacement identifies a protein domain needed for biosynthesis of the sesquiterpene soil odor geosmin. Proc. Natl. Acad. Sci. USA 2003, 100, 1541-1546. [CrossRef]

29. Li, P.; Li, J.; Guo, Z.; Tang, W.; Han, J.; Meng, X.; Hao, T.; Zhu, Y.; Zhang, L.; Chen, Y. An efficient blue-white screening based gene inactivation system for Streptomyces. Appl. Microbiol. Biotechnol. 2015, 99, 1923-1933. [CrossRef]

30. Saitou, N.; Nei, M. The neighbor-joining method: A new method for reconstructing phylogenetic trees. Mol. Biol. Evol. 1987, 4, 406-425.

31. Tanaka, A.; Takano, Y.; Ohnishi, Y.; Horinouchi, S. AfsR recruits RNA polymerase to the afsS promoter: A model for transcriptional activation by SARPs. J. Mol. Biol. 2007, 369, 322-333. [CrossRef]

32. Chater, K.F.; Chandra, G. The use of the rare UUA codon to define "expression space" for genes involved in secondary metabolism, development and environmental adaptation in Streptomyces. J. Microbiol. 2008, 46, 1-11. [CrossRef] [PubMed]

33. Leskiw, B.K.; Lawlor, E.J.; Fernandez-Abalos, J.M.; Chater, K.F. TTA codons in some genes prevent their expression in a class of developmental, antibiotic-negative, Streptomyces mutants. Proc. Natl. Acad. Sci. USA 1991, 88, 2461-2465. [CrossRef] [PubMed]

34. Devine, J.H.; Shadel, G.S.; Baldwin, T.O. Identification of the operator of the lux regulon from the Vibrio fischeri strain ATCC 7744. Proc. Natl. Acad. Sci. USA 1989, 86, 5688-5692. [CrossRef]

35. Ducros, V.M.; Lewis, R.J.; Verma, C.S.; Dodson, E.J.; Leonard, G.; Turkenburg, J.P.; Murshudov, G.N.; Wilkinson, A.J.; Brannigan, J.A. Crystal structure of GerE, the ultimate transcriptional regulator of spore formation in Bacillus subtilis. J. Mol. Biol. 2001, 306, 759-771. [CrossRef] [PubMed]

36. Gambello, M.J.; Iglewski, B.H. Cloning and characterization of the Pseudomonas aeruginosa lasR gene, a transcriptional activator of elastase expression. J. Bacteriol. 1991, 173, 3000. [CrossRef]

37. Stewart, V.; Parales, J., Jr.; Merkel, S.M. Structure of genes narL and narX of the nar (nitrate reductase) locus in Escherichia coli K-12. J. Bacteriol. 1988, 170, 1589-1597. [CrossRef]

38. Henikoff, S.; Wallace, J.C.; Brown, J.P. Finding protein similarities with nucleotide sequence databases. Methods Enzymol. 1989, 183, 111-132.

39. Fuqua, W.; Winans, S.C.; Greenberg, E.P. Quorum sensing in bacteria: The LuxR-LuxI family of cell density-responsive transcriptional regulators. J. Bacteriol. 1994, 176, 269-275. [CrossRef]

40. Knirschová, R.; Nováková, R.; Fecková, L.; Timko, J.; Turna, J.; Bistáková, J.; Kormanec, J. Multiple regulatory genes in the salinomycin biosynthetic gene cluster of Streptomyces albus CCM 4719. Folia Microbiol. 2007, 52, 359-365. [CrossRef]

41. Santos-Aberturas, J.; Vicente, C.M.; Guerra, S.M.; Payero, T.D.; Martín, J.F.; Aparicio, J.F. Molecular control of polyene macrolide biosynthesis: Direct binding of the regulator PimM to eight promoters of pimaricin genes and identification of binding boxes. J. Biol. Chem. 2011, 286, 9150-9161. [CrossRef]

42. Zhang, P.; Zhao, Z.; Li, H.; Chen, X.L.; Deng, Z.; Bai, L.; Pang, X. Production of the antibiotic FR-008/Candicidin in Streptomyces sp. FR-008 is coregulated by two regulators, FscRI and FscRIV, from different transcription factor families. Microbiology 2015, 161, 539. [CrossRef] [PubMed]

43. Choi, S.H.; Greenberg, E.P. The C-terminal region of the Vibrio fischeri LuxR protein contains an inducer-independent lux gene activating domain. Proc. Natl. Acad. Sci. USA 1991, 88, 11115-11119. [CrossRef] [PubMed]

44. Zheng, Q.; Gao, S. The effect of surfactant on fermentation of kitasamycin in Streptomyces kitasatoensis. Biotechnol. Appl. Biochem. 2016, 63, 895-900. [CrossRef] [PubMed]

45. Kirst, H.A.; Wild, G.; Baltz, R.; Seno, E.; Hamill, R.; Paschal, J.; Dorman, D. Elucidation of structure of novel macrolide antibiotics produced by mutant strains of Streptomyces fradiae. J. Antibiot. 1983, 36, 376-382. [CrossRef]

46. Horinouchi, S.; Kito, M.; Nishiyama, M.; Furuya, K.; Hong, S.-K.; Miyake, K.; Beppu, T. Primary structure of AfsR, a global regulatory protein for secondary metabolite formation in Streptomyces coelicolor A3 (2). Gene 1990, 95, 49-56. [CrossRef]

47. Horinouchi, S. AfsR as an integrator of signals that are sensed by multiple serine/threonine kinases in Streptomyces coelicolor A3 (2). J. Ind. Microbiol. Biotechnol. 2003, 30, 462-467. [CrossRef]

48. Maharjan, S.; Oh, T.-J.; Lee, H.C.; Sohng, J.K. Identification and functional characterization of an afsR homolog regulatory gene from Streptomyces venezuelae ATCC 15439. J. Microbiol. Biotechnol. 2009, 19, 121-127. 
49. Seipke, R.F.; Song, L.; Bicz, J.; Laskaris, P.; Yaxley, A.M.; Challis, G.L.; Loria, R. The plant pathogen Streptomyces scabies 87-22 has a functional pyochelin biosynthetic pathway that is regulated by TetR-and AfsR-family proteins. Microbiology 2011, 157, $2681-2693$. [CrossRef]

50. Wang, W.; Wang, H.; Hu, H.; Peng, H.; Zhang, X. Overexpression of afsR and optimization of metal chloride to improve lomofungin production in Streptomyces lomondensis S015. J. Microbiol. Biotechnol. 2015, 25, 672-680. [CrossRef]

51. Wilson, D.J.; Xue, Y.; Reynolds, K.A.; Sherman, D.H. Characterization and analysis of the PikD regulatory factor in the pikromycin biosynthetic pathway of Streptomyces venezuelae. J. Bacteriol. 2001, 183, 3468-3475. [CrossRef]

52. Hur, Y.-A.; Choi, S.-S.; Sherman, D.H.; Kim, E.-S. Identification of TmcN as a pathway-specific positive regulator of tautomycetin biosynthesis in Streptomyces sp. CK4412. Microbiology 2008, 154, 2912-2919. [CrossRef] [PubMed]

53. Kuščer, E.; Coates, N.; Challis, I.; Gregory, M.; Wilkinson, B.; Sheridan, R.; Petković, H. Roles of rapH and rapG in positive regulation of rapamycin biosynthesis in Streptomyces hygroscopicus. J. Bacteriol. 2007, 189, 4756-4763. [CrossRef]

54. Intra, B.; Euanorasetr, J.; Nihira, T.; Panbangred, W. Characterization of a gamma-butyrolactone synthetase gene homologue $($ stcA) involved in bafilomycin production and aerial mycelium formation in Streptomyces sp. SBI034. Appl. Microbiol. Biotechnol. 2016, 100, 2749-2760. [CrossRef] [PubMed]

55. Aroonsri, A.; Kitani, S.; Hashimoto, J.; Kosone, I.; Izumikawa, M.; Komatsu, M.; Fujita, N.; Takahashi, Y.; Shinya, K.; Ikeda, H. Pleiotropic control of secondary metabolism and morphological development by KsbC, a butyrolactone autoregulator receptor homologue in Kitasatospora setae. Appl. Environ. Microb. 2012, 78, 8015-8024. [CrossRef] [PubMed]

56. Choi, S.-U.; Lee, C.-K.; Hwang, Y.-I.; Kinoshita, H.; Nihira, T. Cloning and functional analysis by gene disruption of a gene encoding a $\gamma$-butyrolactone autoregulator receptor from Kitasatospora setae. J. Bacteriol. 2004, 186, 3423-3430. [CrossRef] [PubMed]

57. Kumar, S.; Stecher, G.; Tamura, K. MEGA7: Molecular Evolutionary Genetics Analysis version 7.0 for bigger datasets. Mol. Biol. Evol. 2016, 33, 1870-1874. [CrossRef]

58. Livak, K.J.; Schmittgen, T.D. Analysis of relative gene expression data using real-time quantitative PCR and the $2^{-\Delta \Delta C T}$ method. Methods 2001, 25, 402-408. [CrossRef] 